\title{
SAKK 24/09: safety and tolerability of bevacizumab plus paclitaxel vs. bevacizumab plus metronomic cyclophosphamide and capecitabine as first-line therapy in patients with HER2- negative advanced stage breast cancer - a multicenter, randomized phase III trial
}

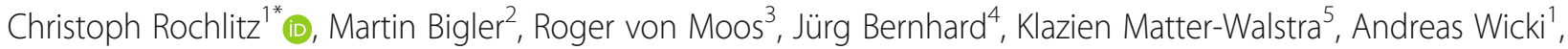
Khalil Zaman ${ }^{6}$, Sandro Anchisi ${ }^{7}$, Marc Küng ${ }^{8}$, Kyung-Jae Na $^{2,9}$, Daniela Bärtschi ${ }^{2}$, Markus Borner ${ }^{10}$, Tamara Rordorf ${ }^{11}$, Daniel Rauch ${ }^{12}$, Andreas Müller ${ }^{13}$, Thomas Ruhstaller ${ }^{14}$, Marcus Vetter ${ }^{1}$, Andreas Trojan ${ }^{15}$, Ursula Hasler-Strub ${ }^{14}$, Richard Cathomas ${ }^{3}$, and Ralph Winterhalder ${ }^{16}$ on behalf of the Swiss Group for Clinical Cancer Research (SAKK)

\footnotetext{
Abstract

Background: Adding bevacizumab to chemotherapy improves response rates and progression-free survival (PFS) in metastatic breast cancer ( $\mathrm{mBC}$ ). We aimed to demonstrate decreased toxicity with metronomic chemotherapy/ bevacizumab compared with paclitaxel/bevacizumab.

Methods: This multicenter, randomized phase III trial compared bevacizumab with either paclitaxel (arm A) or daily oral capecitabine-cyclophosphamide (arm B) as first-line treatment in patients with HER2-negative advanced breast cancer. The primary endpoint was the incidence of selected grade 3-5 adverse events (AE) including: febrile neutropenia, infection, sensory/motor neuropathy, and mucositis. Secondary endpoints included objective response rate, disease control rate, PFS, overall survival (OS), quality of life (QoL), and pharmacoeconomics. The study was registered prospectively with ClinicalTrials.gov, number NCT01131195 on May 25, 2010.

Results: Between September 2010 and December 2012, 147 patients were included at 22 centers. The incidence of primary endpoint-defining AEs was similar in arm A (25\% [18/71]; $95 \%$ Cl 15-35\%) and arm B (24 \% [16/68]; $95 \%$ $\mathrm{Cl} 13-34 \% ; P=0.96)$. Objective response rates were $58 \%(42 / 73 ; 95 \% \mathrm{Cl} 0.46-0.69)$ and $50 \%(37 / 74 ; 95 \% \mathrm{Cl} 0.39-$ $0.61)$ in arms $A$ and $B$, respectively $(P=0.45)$. Median PFS was 10.3 months $(95 \% \mathrm{Cl} 8.7-11.3)$ in arm $\mathrm{A}$ and 8. 5 months $(95 \% \mathrm{Cl} 6.5-11.9)$ in arm $\mathrm{B}(P=0.90)$. Other secondary efficacy endpoints were not significantly different between study arms. The only statistically significant differences in QoL were less hair loss and less numbness in arm B. Treatment costs between the two arms were equivalent.

(Continued on next page)
}

\footnotetext{
* Correspondence: Christoph.Rochlitz@usb.ch

'Department of Oncology, University Hospital Basel, Petersgraben 4, Basel

CH-4031, Switzerland

Full list of author information is available at the end of the article
} 
(Continued from previous page)

Conclusion: This trial failed to meet its primary endpoint of a reduced rate of prespecified grade 3-5 AEs with metronomic bevacizumab, cyclophosphamide and capecitabine.

Keywords: Metronomic chemotherapy, Bevacizumab, Breast cancer, Toxicity

\section{Background}

While there is no generally accepted, optimal first-line chemotherapy regimen for $\mathrm{mBC}$, most experts favor the use of taxanes and anthracyclines, either as monotherapy or in different two-drug combinations. Combination regimens typically achieve superior response rates (RR) and longer PFS than mono-chemotherapy, but have limited impact on OS [1-4] and are associated with increased toxicity [5].

The E2100 randomized phase III trial reported a near doubling of PFS and RR with paclitaxel plus bevacizumab compared with paclitaxel alone in patients with $\mathrm{mBC}$ [6]. Increased rates of sensory neuropathy, febrile neutropenia and infection, and severe fatigue occurred with combination therapy. These data led to the FDA (Food and drug administration) granting accelerated approval to bevacizumab plus weekly paclitaxel for the first-line treatment of HER2-negative $\mathrm{mBC}$ in 2008. However, this approval was later removed because: additional randomized trials $[7,8]$ showed less pronounced PFS and RR benefits; no trial demonstrated an OS benefit (later confirmed in meta-analyses $[9,10])$; and additional safety concerns were discussed [11].

Metronomic chemotherapy is the frequent administration of chemotherapy at low, minimally-toxic doses with no prolonged drug-free intervals [12]. No trial has directly compared bevacizumab plus paclitaxel with bevacizumab plus metronomic cyclophosphamide-capecitabine. However, between-trial comparisons suggest that these regimens have similar efficacy but that toxicity with metronomic therapy is substantially reduced $[6,13]$. We therefore designed a randomized phase III trial to test if metronomic chemotherapy plus bevacizumab decreases high-grade toxicity compared with paclitaxel plus bevacizumab.

\section{Methods}

\section{Patients}

Eligible patients had cytologically/histologically proven metastatic or locally recurrent inoperable HER2-negative breast cancer evaluable according to RECIST v1.1 criteria [14]. Other inclusion criteria included WHO (World Health Organization) performance status 0-2, low-risk for bleeding, and available baseline QoL and pharmacoeconomic assessment. Patients with (neo)adjuvant chemotherapy within the previous 6 months (12 months for taxane- and 5-FU-based chemotherapy), anti-VEGF (vascular endothelial growth factor) therapy within the previous 12 months, or prior chemotherapy for metastatic or locally advanced/recurrent breast cancer were excluded.
Other exclusion criteria comprised known CNS (Central Nervous System) metastases; severe cardiovascular, renal, hepatic, or neurological disease; and history of abdominal fistula, gastrointestinal perforation, or intra-abdominal abscess.

\section{Trial design}

This multicenter, randomized parallel open-label phase III trial compared bevacizumab plus paclitaxel versus bevacizumab plus metronomic capecitabine-cyclophosphamide as first-line therapy in patients with HER2-negative metastatic or locally recurrent breast cancer.

Patients received $10 \mathrm{mg} / \mathrm{kg}$ i.v. bevacizumab every 2 weeks with either $90 \mathrm{mg} / \mathrm{m}^{2}$ i.v. paclitaxel (days $1 / 8 / 15$ of a 4 week cycle; arm A [6]) or daily oral $50 \mathrm{mg}$ cyclophosphamide and $3 \times 500 \mathrm{mg}$ capecitabine (arm B [13]). All medications were given until disease progression (PD), unacceptable $\mathrm{AE}$ according to local investigator's assessment, or consent withdrawal. After occurrence of an unacceptable $\mathrm{AE}$ to one drugs, the remaining tolerated drug(s) was (were) given until PD, consent withdrawal, or unacceptable AE.

Treatments were assigned online (www.sakk.ch/sinatras). Randomization (1:1) using minimization was stratified according to measurable/evaluable disease, $\mathrm{WHO}$ performance status (0/1 vs. 2$)$, and center.

\section{Endpoints}

The primary endpoint was the incidence of pre-specified grade 3-5 AEs $(\mathrm{CTCAE}=$ Common Terminology Criteria for Adverse Events, version 4.0) occurring during the trial or within 30 days of last treatment, regardless of the causal relationship to the trial drugs. These comprised: any AEs of the system/organ classes infection and infestation, febrile neutropenia, nausea, vomiting, oral mucositis, peripheral sensory neuropathy, arthralgia, myalgia, headache, thromboembolic events, cerebrovascular ischemia, left ventricular systolic dysfunction; any AEs correlated with bleeding; or any gastrointestinal perforation.

Palmar-plantar erythrodysesthesia syndrome (hand-foot syndrome, HFS), a key toxicity of capecitabine, was not a primary endpoint-defining $\mathrm{AE}$ in our protocol as grade 3/ 4 HFS was not previously observed with metronomic cyclophosphamide-capecitabine plus bevacizumab [13]. To exclude a potential bias caused by this omission we performed an exploratory sensitivity analysis that included HFS grade $\geq 3$ as an event. 
Secondary endpoints included: objective response rate, disease control rate, PFS, OS, other AEs, QoL, and pharmacoeconomics.

The primary QoL endpoint was physical well-being measured by self-assessment questionnaire at baseline (pre-randomization) and at monthly clinic visits for the first 12 months until PD. The questionnaire comprised indicators of physical well-being, mood, coping effort, overall treatment burden, health perception, appetite, tiredness, hair loss, nausea/vomiting, and numbness/tingling in hands/feet [15-20]. Higher scores (range 0-100) reflected a better condition. A change from baseline in physical well-being of $\geq 6$ points was defined as clinically meaningful [21]. An improvement of this magnitude maintained for $\geq 12$ consecutive weeks was defined as QoL benefit.

\section{Health economic analysis (HEA)}

The primary endpoint of the HEA was the total incurred treatment costs until patients stopped trial treatment. The HEA adopted a health system perspective including all substantial direct medical costs incurred in the treatment of the patient. Health-related QoL was measured using EQ-5D (EuroQOL five dimensions questionnaire).

\section{Statistical methods}

Sample size was estimated for the primary endpoint using East 5.0 and adjusted with the Casagrande-Pike-Smith correction. The expected incidence rate of predefined grade 3-5 AEs was $30 \%$ in arm A and $10 \%$ in arm B. With a two-sided $5 \%$ type I error probability and $80 \%$ power, 71 evaluable patients per arm were required. The primary endpoint was analyzed in all evaluable patients (patients who received the first bevacizumab administration of the second cycle or who experienced a primary endpoint-defining event), secondary exploratory efficacy endpoints were analyzed among all randomized patients, and secondary exploratory safety endpoints were analyzed among all randomized patients who received $\geq 1$ dose of trial medication. All analyses were conducted using SAS 9.2 and $\mathrm{R}$ 3.0.0.

$95 \%$ confidence intervals were calculated for rates and arms were compared using two-sided z-tests with pooled variance and continuity correction. Time-to-event data were analyzed by Kaplan-Meier analyses and compared using log-rank tests or Gray-Tsiatis tests for cure models.

The effects of treatment allocation and time on QoL scores were estimated using mixed linear models for repeated measurements (without controlling for multiple testing).

Differences in treatment costs between arms in the HEA were tested by the Wilcoxon rank-sum test. A global multivariable linear model (proc genmod) with a gamma distribution and a logarithmic link function was used to analyze costs after controlling for age.

\section{Results}

From September 2010 through December 2012, we randomized 147 patients to bevacizumab plus paclitaxel $(N=73$; arm A) or metronomic therapy $(N=74$; arm B) at 22 SAKK centers in Switzerland (Fig. 1). Patient characteristics are listed in Table 1.

\section{Safety}

The primary endpoint was evaluable in 139 patients. A primary endpoint-defining AE occurred in 18/71(25\%; $95 \%$ CI 15-35\%) patients in arm A and 16/68 (24\%; $95 \%$ CI 13-34 \%) in arm B $(P=0.96)$. These AEs comprised neuropathy $(N=7)$, infection $(N=5)$, thromboembolic events $(N=3)$, arthralgia $(N=2)$, and nausea $(N$ $=1)$ in arm $\mathrm{A}$, and infection $(N=4)$, thromboembolic events $(N=4)$, nausea $(N=3)$, arthralgia $(N=2)$, headache $(N=2)$, and mucositis $(N=1)$ in arm B. Only one of these AEs was grade 4 (a thromboembolic event in arm A).

Results were similar in the sensitivity analysis that included HFS as a primary endpoint-defining AE: 18 (25\%) patients in arm A and $19(28 \%)$ in arm B had an event $(P=0.88)$.

Seventeen patients stopped treatment because of unacceptable toxicities: $12(17 \%)$ in arm A and five (7 \%) in arm B. The main toxicities leading to suspension or delay were neuropathy, neutropenia, leukopenia, fatigue, and infection for paclitaxel; HFS, neutropenia, nausea/vomiting, and loss of appetite for capecitabine; and vomiting/nausea, thrombocytopenia, diarrhea, and neutropenia for cyclophosphamide.

\section{Efficacy}

Objective RRs were 58 \% (42/73; $95 \%$ CI 46-69\%) and $50 \%(37 / 74 ; 95 \%$ CI 39-61 \%) in arms A and B, respectively $(P=0.45)$. Four and two patients in arms $\mathrm{A}$ and $\mathrm{B}$, respectively, achieved a complete response. Disease control rates were similar between arms (79 \% [95 \% CI 70-89\%] in arm A and 64\% [95 \% CI 53-74\%] in arm B).

At data cutoff, 103 patients had progressed. Median PFS did not differ between study arms $(P=0.83$; Table 2 and Fig. 2).

Seventy-two patients died during a median 26.1 months follow-up (Table 2). OS was numerically higher in arm A vs arm $\mathrm{B}(P=0.24)$.

\section{QoL}

QoL forms were available for $82 \%$ of patients. Patients in arm B reported substantially less hair loss $(p<0.0001$; Fig. 3) and less numbness with increasing time $(p<0.01)$ than those in arm A, and a tendency toward less overall treatment burden $(P=0.11)$. Over the first 12 months 


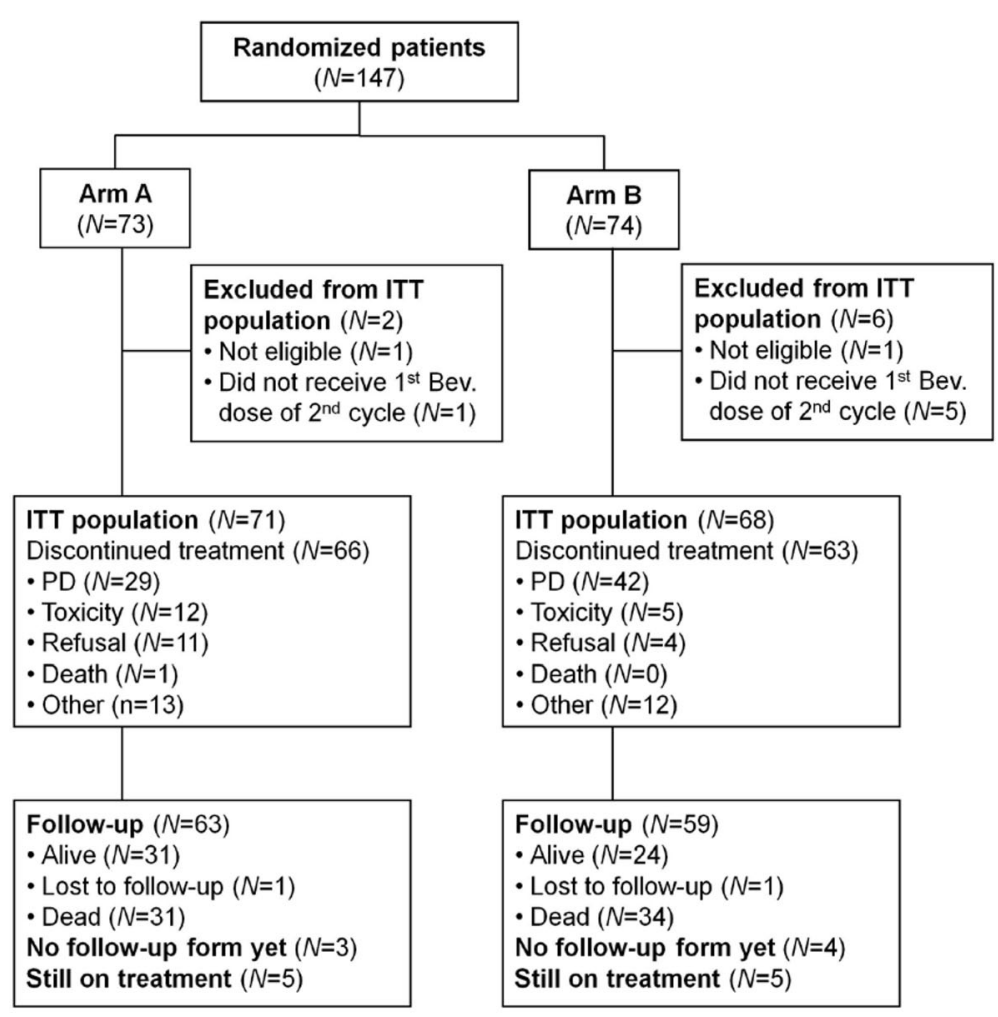

Bev=bevacizumab; PD=progressive disease

Fig. 1 CONSORT diagram. The flow diagram shows the intention-to-treat population of 147 patients included in the primary analysis of bevacizumab plus paclitaxel (arm A) compared with bevacizumab plus metronomic chemotherapy (arm B). Bev=bevacizumab;

$P D=$ progressive disease

treatment, 14/70 (20\%) patients in arm $\mathrm{A}$ and 14/73 (19 \%) in arm B indicated a QoL benefit of a similar median duration (140 and 139 days, respectively).

\section{HEA}

By January 2014, 129 patients had stopped trial treatment (Table 3). There was no cost advantage for bevacizumab plus metronomic chemotherapy over bevacizumab plus paclitaxel. Mean total incurred treatment costs were $\mathrm{CHF}$ (Swiss Francs) 69,474 ( US\$75,000) for arm A and CHF 80,324 (US\$86,600) for arm B. Age did not significant effect the results.

\section{Discussion}

We evaluated a combination of metronomic chemotherapy and bevacizumab that was described as effective and almost non-toxic in a small phase II trial [13]. We focused on grade $3 / 4$ toxicities that are associated with a decrease in QoL and considerable additional treatment costs.

We could not demonstrate a superior toxicity profile of bevacizumab plus metronomic chemotherapy compared with bevacizumab plus paclitaxel. However, there seem to have been some qualitative differences in terms of toxicity. For instance, no grade 3/4 neuropathy was observed in the metronomic arm, whereas this adverse event occurred in almost $10 \%$ of patients treated with paclitaxel. This toxicity is well-known to be associated with all taxanes, and especially with paclitaxel when given in a weekly regimen. Furthermore, we observed less treatment interruptions due to unacceptable toxicity in the metronomic (7\%) versus the paclitaxel arm (17\%). Although these differences were not statistically significant due to lack of power, it is highly likely that a larger study would have shown a significantly lower rate of at least some of these toxicities in the metronomic arm of the study.

The high rate of primary endpoint-defining grade $3 / 4$ AEs in the metronomic arm of our trial is difficult to explain. These occurred in $24 \%$ of patients in our trial but were absent in the previous phase II trial [13]. This finding might reflect differences between study populations or differences in reporting of AEs, due to the specific focus on these events in our trial. One quarter of paclitaxel-treated patients in our trial experienced 
Table 1 Characteristics of patients in the primary analysis according to chemotherapy cohort

\begin{tabular}{|c|c|c|}
\hline Characteristic & $\begin{array}{l}\text { Arm A } \\
(N=71)\end{array}$ & $\begin{array}{l}\text { Arm B } \\
(N=68)\end{array}$ \\
\hline Age - median (range) & $64(30-82)$ & $62(29-81)$ \\
\hline \multicolumn{3}{|l|}{ WHO performance status, n (\%) } \\
\hline 0,1 & $66(93.0 \%)$ & $64(94.1 \%)$ \\
\hline 2 & $5(7.0 \%)$ & $4(5.9 \%)$ \\
\hline Previous (neo-)adjuvant chemotherapy, n (\%) & $38(53.5 \%)$ & $37(54.4 \%)$ \\
\hline Previous taxane-based chemotherapy, n (\%) & $21(29.6 \%)$ & $20(29.4 \%)$ \\
\hline \multicolumn{3}{|l|}{ Estrogen-receptor status, n (\%) } \\
\hline negative & $10(14.1 \%)$ & $15(22.1 \%)$ \\
\hline positive & $61(85.9 \%)$ & $52(76.5 \%)$ \\
\hline unknown & & $1(1.5 \%)$ \\
\hline \multicolumn{3}{|l|}{ Progesterone-receptor status, n (\%) } \\
\hline negative & $23(32.4 \%)$ & $27(39.7 \%)$ \\
\hline positive & $48(67.6 \%)$ & $40(58.8 \%)$ \\
\hline unknown & & $1(1.5 \%)$ \\
\hline \multicolumn{3}{|l|}{ Disease evaluation, n (\%) } \\
\hline Evaluable & $13(18.3 \%)$ & $8(11.8 \%)$ \\
\hline Measurable & $58(81.7 \%)$ & $60(88.2 \%)$ \\
\hline \multicolumn{3}{|l|}{ Presence of metastases, $\mathrm{n}(\%)$} \\
\hline Liver & $41(57.7 \%)$ & $37(54.4 \%)$ \\
\hline Lung & $25(35.2 \%)$ & $33(48.5 \%)$ \\
\hline Bone & $52(73.2 \%)$ & 49 (72.1 \%) \\
\hline Brain & $0(0 \%)$ & $0(0 \%)$ \\
\hline Soft-tissue & 12 (16.9\%) & $8(11.8 \%)$ \\
\hline Other & 21 (29.6 \%) & 13 (19.1\%) \\
\hline
\end{tabular}

primary endpoint-defining AEs, close to the incidence seen in E2100 (36 \%) [6], and in more recent studies of bevacizumab in breast cancer [22]. We therefore assume that our patient population was not substantially different from that of other studies [13].

Table 2 Progression free (PFS) and overall survival (OS)

\begin{tabular}{lll}
\hline & Arm A (N=73) & Arm B (N=74) \\
\hline PFS & $49(67.1 \%)$ & $54(73.0 \%)$ \\
Events, n (\%) & $24(32.9 \%)$ & $20(27.0 \%)$ \\
Censored, n (\%) & $10.3(8.7,11.4)$ & $8.5(6.5,11.9)$ \\
Median (95\% Cl), months & 0.83 & \\
Log-rank test $P$-value & & \\
OS & $33(45.2 \%)$ & $35(52.7 \%)$ \\
Events, $\mathrm{n}(\%)$ & $40(54.8 \%)$ & $18.7(14.6, \mathrm{NA})$ \\
Censored, $\mathrm{n}(\%)$ & $25.6(18.9, \mathrm{NA})$ & \\
Median (95 \% Cl), months & 0.24 & \\
Log-rank test $P$-value & &
\end{tabular}

Cl confidence interval
The PFS of 10.3 months observed in the taxane arm of our trial is in line with other randomized trials of first-line taxane/bevacizumab combinations (range 9.2-11.3 months) [6-8]. The PFS in the metronomic arm of our trial (8.5 months) is comparable to that reported in the phase II trial (10.5 months), though this trial also included patients in subsequent lines of therapy for advanced breast cancer [13]. Similarly, the RRs in our trial (paclitaxel arm: $58 \%$; metronomic arm: $50 \%$ ) are consistent with RRs in the trials upon which our study was based (E2100/RIBBON1: 37$51 \%$; Dellapasqua et al: $48 \%[6,8,13])$. Interestingly, a small phase II trial in 26 patients using the same metronomic therapy protocol (capecitabine, cyclophosphamide, and bevacizumab) in the same patient population (HER-2 negative $\mathrm{mBC}$ ) as in our trial, but adding erlotinib $100 \mathrm{mg}$ daily, reported a response rate of $62 \%$ and a time to progression of 10 months compatible with but by no means proving a possible additional benefit of EGFR-inhibition in this population [23].

The QoL benefit based on patient-reported outcomes was comparable between arms. However, the reduced incidence of alopecia and numbness seen with metronomic chemotherapy might still make this regimen attractive to a substantial number of patients. The HEA failed to demonstrate a cost benefit of bevacizumab plus metronomic chemotherapy over bevacizumab plus paclitaxel. While treatment costs were not significantly different between study arms, mean (but not median) total costs were surprisingly higher when bevacizumab was combined with oral metronomic therapy versus paclitaxel. This finding is somewhat surprising on the background of previous studies and a recent review suggesting metronomic chemotherapy as a low-cost, well-tolerated, and easy to access strategy even in resource-limited countries [24].

It is tempting to speculate that both, the lack of benefit in QoL and the relatively high treatment costs in the metronomic compared to the standard arm are due to the unexpectedly high level of toxicity seen with metronomic chemotherapy in our trial.

Another possible explanation of the somewhat surprising results of our study could be subtle differences in the study populations, with for instance lung metastases being over-represented in the metronomic arm, while soft-tissue and other types of metastases appear to be more frequent in the paclitaxel arm. The randomization of patients was stratified according to measurable versus evaluable disease, performance status $0 / 1$ versus 2 , and center, which lead to a balanced distribution of these potentially prognostic/predictive factors between the two study arms. However, because of the relatively small number of cases in the trial it is impossible to determine the influence of additional factors such as the localization of metastases on our results. 


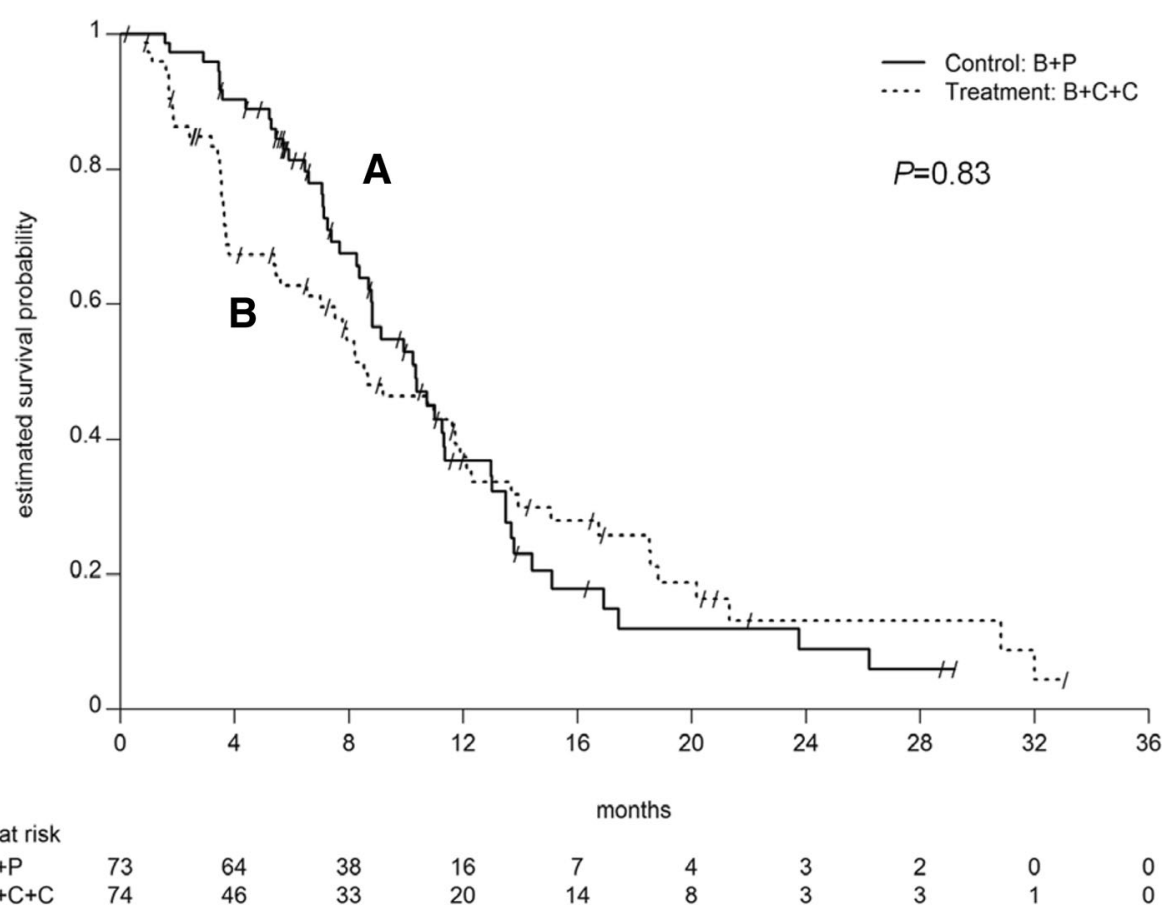

Fig. 2 Progression free survival according to treatment arm. The continuous line indicates the standard arm (a), the dashed line the experimental arm (b)
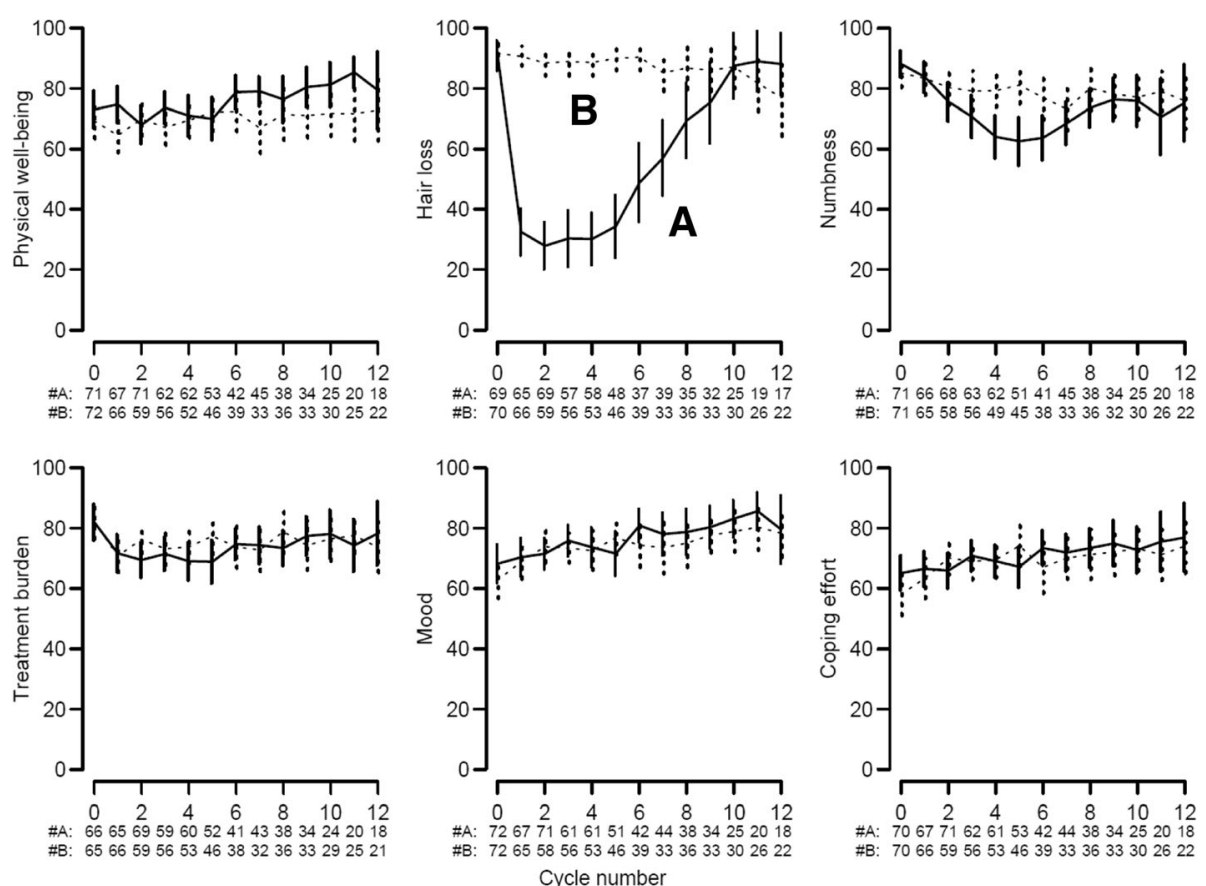

Fig. 3 Physical well-being, hair loss, numbness, treatment burden, mood and coping effort. Data are means with $95 \%$ Cls from baseline over 12 treatment cycles with the number of patients for each cycle. Higher scores indicate better condition. The continuous lines indicate the standard arm (a), the dashed lines the experimental arm (b) 
Table 3 Health economic analysis

\begin{tabular}{|c|c|c|c|c|}
\hline & Mean & $95 \%$ Cl for mean & Median & SD \\
\hline \multicolumn{5}{|c|}{ Arm A: Bevacizumab + paclitaxel $(N=66)$} \\
\hline TTS, months & 7.3 & $6.3-8.2$ & 5.9 & 4.0 \\
\hline Utility & 0.8 & $0.7-0.8$ & 0.8 & 0.2 \\
\hline QALM & 5.9 & $5.0-6.8$ & 5.1 & 3.7 \\
\hline Total incurred costs, CHF & 69,474 & $60,624-78,324$ & 61,815 & 36,001 \\
\hline Costs per month, CHF & 10,044 & $9,216-10,871$ & 9,385 & 3,367 \\
\hline Costs per QALM & 14,389 & $11,955-16,823$ & 11,249 & 9,900 \\
\hline \multicolumn{5}{|c|}{ Arm B: Bevacizumab + capecitabine + cyclophosphamide $(N=63)$} \\
\hline TTS, months & 8.5 & $6.7-10.2$ & 6.8 & 7.0 \\
\hline Utility & 0.7 & $0.7-0.8$ & 0.7 & 0.2 \\
\hline QALM & 6.5 & $5.0-8.1$ & 5.0 & 6.3 \\
\hline Total incurred costs, CHF & 80,324 & $62,975-97,672$ & 61,751 & 68,885 \\
\hline Costs per month, CHF & 10,229 & $9,317-11,142$ & 9,158 & 3,622 \\
\hline Costs per QALM & 40,826 & $-7,257-88,909$ & 12,025 & 190,922 \\
\hline
\end{tabular}

$N$ number of patients, $T S$ time to treatment stop, QALM quality adjusted life month, CHF swiss francs, $\mathrm{Cl}$ confidence interval, $S D$ standard deviation of the mean

Unit costs for the health economics analysis are provided in the Additional file 1: Table S1

\section{Conclusions}

In conclusion, we were unable to achieve a better safety and efficacy profile for bevacizumab by combining with metronomic chemotherapy compared with paclitaxel. We therefore do not recommend further testing of this regimen of bevacizumab plus metronomic chemotherapy in $\mathrm{mBC}$.

\section{Key message}

Combining bevacizumab with metronomic cyclophospha mide-capecitabine does not achieve a superior safety and efficacy profile compared with bevacizumab plus paclitaxel in patients with advanced HER2 (human epidermal growth factor receptor 2)-negative breast cancer.

\section{Additional file}

Additional file 1: Table S1. Unit costs for the health economics analysis. (DOCX $16 \mathrm{~kb})$

\section{Abbreviations}

AE: Adverse events; B + C + C: Bevacizumab + cyclophosphamide + capecitabine; B + P: Bevacizumab + paclitaxel; CHF: Swiss franc; $\mathrm{Cl}$ : Confidence interval; CNS: Central nervous system; CTCAE: Common terminology criteria for adverse events; EQ-5D: EuroQOL five dimensions questionnaire; FDA: Food and drug administration; HEA: Health economic analysis; HER2: Human epidermal growth factor receptor 2; HFS: Hand-foot syndrome; ITT: Intention to treat; mBC: Metastatic breast cancer; OS: Overall survival; PD: Progressive disease; PFS: Progression-free survival; QALM: Quality adjusted life month; QoL: Quality of life; RECIST: Response evaluation criteria in solid tumors; RR: Response rates; SAKK: Swiss group for clinical cancer research; SD: Standard deviation of the mean; TTS: Time to treatment stop; US\$: US dollar; VEGF: Vascular endothelial growth factor; WHO: World Health Organization

\section{Acknowledgements}

The final draft of the manuscript was edited by Jamie Ashman of Prism Ideas (funded by the SAKK).

\section{Funding}

The study was partially funded by a grant of the Basel Cancer League and supported by the Swiss State Secretariat for Education, Research and Innovation, and by Roche Pharma (Schweiz) AG. Roche Pharma (Schweiz) AG was involved in trial design, but had no role in data collection, data analysis, data interpretation, or writing of the report. The corresponding author had full access to all the data and the final responsibility to submit for publication.

\section{Availability of data and materials}

The study is registered with ClinicalTrials.gov, number NCT01131195. Registration was done prospectively, before entry of the first patient, on May 25, 2010.

The complete data set is deposited at the Swiss Group for Clinical Cancer Research and available at http://sakk.ch/de/?gclid=CJGGu_bF4c4CFRX gGwod3WWJMA.

\section{Authors' contributions}

CR wrote the study protocol in collaboration with members of the SAKK Coordinating Center, Bern, including MB, DB, K-JN and RW. JB and KM-W were responsible for the QoL and the HEA analysis, respectively. DB collected study data. CR, RvM, KZ, SA, MK, MB, TR, DR, AM, TR, MV, AJ, UH-S, RC, and RW enrolled patients to the study. DB collected study data. MB did the statistical analyses of the study data. CR wrote the manuscript on behalf and with the support of all authors. All authors have approved the manuscript and any revisions.

\section{Competing interests}

Christoph Rochlitz, Consulting or Advisory Role: Roche, Novartis, Astra Zeneca, Amgen, Travel, Accommodations, Expenses: Amgen, Roche, Novartis, Boehringer, Lilly. Martin Bigler, No relationship to disclose. Roger von Moos, Honoraria: Amgen, GSK, Consulting or Advisory Role: Roche, Amgen, MSD, Novartis, Merck-Serono. Jürg Bernhard, No relationship to disclose. Klazien Matter-Walstra, No relationship to disclose. Andreas Wicki, Consulting or Advisory Role: Roche, Novartis, Merck, MSD, Beyer, Amgen. Khalil Zaman, Consulting or Advisory Role: Roche, Novartis, Astra Zeneca, Travel, Accommodations, Expenses: Roche, Novartis, Amgen, Celgene. Sandro Anchisi, Honoraria: Roche, Janssen Cilag AG, Travel, Accommodations, Expenses: Roche. Marc Küng, No relationship to disclose. Kyung-Jae Na, Employment: Novartis, Stock or other ownership: Novartis. Daniela Bärtschi, No relationship to disclose. Markus Borner, No relationship to disclose. Tamara Rordorf, Consulting or Advisory Role: Merck, Travel, Accommodations, Expenses: Roche. Daniel Rauch, No relationship to disclose. Andreas Müller, Honoraria: Roche, Novartis, MSD, Consulting or Advisory Role: Roche, Novartis, Travel, Accommodations, Expenses: Roche, Novartis, Pfizer, Bayer. Thomas Ruhstaller, Consulting or Advisory Role: Roche, Novartis, Expert Testimony: Roche, Travel, Accommodations, Expenses: Roche, Novartis. Marcus Vetter, Consulting or Advisory Role: Roche. Andreas Trojan, No relationship to disclose. Ursula Hasler-Strub, No relationship to disclose. Richard Cathomas, No relationship to disclose. Ralph Winterhalder, No relationship to disclose.

\section{Consent for publication}

Not applicable.

\section{Ethics approval and consent to participate}

This study was approved by the ethics committee of Northwest- and Central Switzerland (= lead ethics committee) and also by 11 additional ethics commmittees all located within Switzerland (see Additional file 1: Table S1). The trial was conducted in accordance with the declaration of Helsinki. Written informed consent was obtained from all patients.

\section{Author details}

${ }^{1}$ Department of Oncology, University Hospital Basel, Petersgraben 4, Basel $\mathrm{CH}-4031$, Switzerland. ${ }^{2}$ SAKK Coordinating Center, Bern, Switzerland. ${ }^{3}$ Department of Oncology, Kantonsspital Graubünden, Chur, Switzerland. ${ }^{4}$ International Breast Cancer Study (IBCSG) and Inselspital, Bern University Hospital, Bern, Switzerland. ${ }^{5}$ SAKK Coordinating Center, Bern, Switzerland and European Center for Pharmaceutical Medicine, University Basel, Basel, Switzerland. ${ }^{6}$ Department of Oncology, University Hospital Lausanne, Lausanne, Switzerland. 'Department of Oncology, Hospital of Valais, Sion, 
Switzerland. ${ }^{8}$ Department of Oncology, Kantonsspital Fribourg, Fribourg, Switzerland. ${ }^{9}$ Present Address: Novartis Pharma, Stein, Switzerland.

${ }^{10}$ Department of Oncology, Spitalzentrum Biel, Biel, Switzerland.

${ }^{11}$ Department of Oncology, University Hospital Zürich, Zürich, Switzerland.

${ }^{12}$ Department of Oncology, Spital STS, Thun, Switzerland. ${ }^{13}$ Department of Oncology, Kantonsspital Winterthur, Winterthur, Switzerland. ${ }^{14}$ Department of Oncology, Kantonsspital St. Gallen, St. Gallen, Switzerland. ${ }^{15}$ Department of Oncology, OnkoZentrum Zürich, Zürich, Switzerland. ${ }^{16}$ Luzerner

Kantonsspital, Luzerne, Switzerland.

Received: 12 January 2016 Accepted: 29 September 2016

Published online: 10 October 2016

\section{References}

1. Albain KS, Nag SM, Calderillo-Ruiz G, et al. Gemcitabine plus paclitaxel versus paclitaxel monotherapy in patients with metastatic breast cancer and prior anthracycline treatment. J Clin Oncol. 2008;26:3950-7.

2. O'Shaughnessy J, Miles D, Vukelja S, et al. Superior survival with capecitabine plus docetaxel combination therapy in anthracyclinepretreated patients with advanced breast cancer: phase III trial results. J Clin Oncol. 2002;20:2812-23.

3. Sledge GW, Neuberg D, Bernardo P, et al. Phase III trial of doxorubicin paclitaxel, and the combination of doxorubicin and paclitaxel as front-line chemotherapy for metastatic breast cancer: an intergroup trial (E1193). J Clin Oncol. 2003;21:588-92.

4. Piccart-Gebhart MJ, Burzykowski T, Buyse M, et al. Taxanes alone or in combination with anthracyclines as first-line therapy of patients with metastatic breast cancer. J Clin Oncol. 2008;26:1980-6.

5. Bernard-Marty C, Cardoso F, Piccart MJ. Facts and controversies in systemic treatment of metastatic breast cancer. Oncologist. 2004;9:617-32.

6. Miller K, Wang M, Gralow J, et al. Paclitaxel plus bevacizumab versus paclitaxel alone for metastatic breast cancer. N Engl J Med. 2007;357:2666-76.

7. Miles DW, Chan A, Dirix LY, et al. Phase III study of bevacizumab plus docetaxel compared with placebo plus docetaxel for the first-line treatment of human epidermal growth factor receptor 2-negative metastatic breast cancer. J Clin Oncol. 2010;28:3239-47.

8. Robert NJ, Diéras V, Glaspy J, et al. RIBBON-1: randomized, double-blind, placebo-controlled, phase III trial of chemotherapy with or without bevacizumab for first-line treatment of human epidermal growth factor receptor 2-negative, locally recurrent or metastatic breast cancer. J Clin Oncol. 2011;29:1252-60.

9. Miles DW, Diéras V, Cortés J, et al. First-line bevacizumab in combination with chemotherapy for HER2-negative metastatic breast cancer: pooled and subgroup analyses of data from 2447 patients. Ann Oncol. 2013;24:2773-80.

10. Valachis A, Polyzos NP, Patsopoulos NA, et al. Bevacizumab in metastatic breast cancer: a meta-analysis of randomized controlled trials. Breast Cancer Res Treat. 2010;122:1-7.

11. Montero AJ, Escobar M, Lopes G, et al. Bevacizumab in the treatment of metastatic breast cancer: friend or foe? Curr Oncol Rep. 2012;14:1-11.

12. Munzone $\mathrm{E}$, Colleoni M. Clinical overview of metronomic chemotherapy in breast cancer. Nat Rev Clin Oncol. 2015;12(11):631-44.

13. Dellapasqua S, Bertolini F, Bagnardi V, et al. Metronomic cyclophosphamide and capecitabine combined with bevacizumab in advanced breast cancer. J Clin Oncol. 2008;26:4899-905.

14. Therasse P, Arbuck SG, Eisenhauer EA, et al. New guidelines to evaluate the response to treatment in solid tumors. European organization for research and treatment of cancer, national cancer institute of the United States, national cancer institute of Canada. J Natl Cancer Inst. 2000;92:205-16.

15. Butow $\mathrm{P}$, Coates $\mathrm{A}$, Dunn $\mathrm{S}$, et al. On the receiving end. IV: validation of quality of life indicators. Ann Oncol. 1991;2:597-603.

16. Bernhard J, Sullivan M, Hürny $C$, et al. Clinical relevance of single item quality of life indicators in cancer clinical trials. Br J Cancer. 2001;84:1156-65.

17. Hürny C, Bernhard J, Bacchi M, et al. The perceived adjustment to chronic illness scale (PACIS): a global indicator of coping for operable breast cancer patients in clinical trials. Swiss group for clinical cancer research (SAKK) and the international breast cancer study group (IBCSG). Support Care Cancer. 1993;1:200-8.

18. Bernhard J, Maibach R, Thürlimann B, et al. Patients' estimation of overall treatment burden: why not ask the obvious? J Clin Oncol. 2002;20:65-72.

19. Hürny $C$, van Wegberg B, Bacchi M, et al. Subjective health estimations (SHE) in patients with advanced breast cancer: an adapted utility concept for clinical trials. Br J Cancer. 1998;77:985-91.
20. Coates A, Glasziou P, McNeil D. On the receiving end-III. Measurement of quality of life during cancer chemotherapy. Ann Oncol. 1990;1:213-7.

21. Bernhard J, Thürlimann B, Schmitz SF, et al. Defining clinical benefit in postmenopausal patients with breast cancer under second-line endocrine treatment: does quality of life matter? J Clin Oncol. 1999;17:1672-9.

22. Cortes J, Calvo V, Ramirez-Merino N, et al. Adverse events risk associated with bevacizumab addition to breast cancer chemotherapy: a meta-analysis. Ann Oncol. 2012;23:1130-7.

23. Montagna E, Cancello G, Bagnardi V, et al. Metronomic chemotherapy combined with bevacizumab and erlotinib in patients with metastatic HER2-negative breast cancer: clinical and biologicalactivity. Clin Breast Cancer. 2012;12(3):207-14.

24. André N, Banavali S, Snihur Y, Pasquier E. Has the time come for metronomics in low-income and middle-income countries? Lancet Oncol. 2013;14(6):e239-48.

\section{Submit your next manuscript to BioMed Central and we will help you at every step:}

- We accept pre-submission inquiries

- Our selector tool helps you to find the most relevant journal

- We provide round the clock customer support

- Convenient online submission

- Thorough peer review

- Inclusion in PubMed and all major indexing services

- Maximum visibility for your research

Submit your manuscript at www.biomedcentral.com/submit
Biomed Central 